\title{
ANALYSIS OF PATIENTS FLOW IN AN EMERGENCY WARD USING FUZZY CRITICAL PATH METHOD AND FUZZY PROGRAM EVALUTION AND REVIEW TECHNIQUE (A CASE STUDY OF AMINU KANO TEACHING HOSPITAL, KANO, NIGERIA)
}

\author{
Ali Maianguwa Shuaibu and Maryam Nuraini Muhammad \\ Department of Mathematics, Federal University Dutse, Jigawa State, Nigeria \\ Corresponding Author's Email: maths@ fud.edu.ng
}

\begin{abstract}
In this paper, a mathematical model is proposed to handle project scheduling problem under vagueness. This is done by assuming the durations of activities to be imprecise and represented by trapezoidal fuzzy numbers. In particular, the paper aimed to model patients flow process in imprecise or vague circumstances using critical path analysis. Furthermore, the research discusses utilization of fuzzy critical path method in conjunction with fuzzy program evaluation and review technique to determine the duration required for the treatment of patients in emergency ward of Aminu Kano teaching hospital. The activities considered are those affecting patients flow during treatments in the emergency ward and the related project network is drawn. The reason for incorporating fuzzy logic in this research comes from the fact that fuzzy numbers are more reliable in dealing with ambiguous processes such as witnessed in the emergency ward of a hospital. The choice of emergency department for this work is because it is a major access point where from the beginning patients are given treatments for a wide range of sicknesses and injuries. These sicknesses and injuries may be deadly and requires prompt attention. Therefore, any delay in patient treatment in the emergency ward may result in undesired consequences and losses of lives.
\end{abstract}

Findings through the application of fuzzy CPM and fuzzy PERT enabled the determination of the critical path, slack times and patients' treatment expected completion time. The entire idea is to reduce unnecessary delay, eliminate unnecessary activities at the same time increase the quality of treatment of patients in the hospital.

Keywords: Emergency ward, fuzzy critical path, patient treatment, fuzzy logic, fuzzy program evaluation and review technique.

\section{INTRODUCTION}


A project is a unique set of tasks with defined objectives, precedence relationships, numerous activities and defined period for completion of the tasks (Gül, Güneri \& Güneş, 2017). Project management deals with the conception, feasibility analysis, planning, execution, monitoring, control, and termination of a project (Reid \& Sanders, 2010). It includes stabilizing the contending desires for time, cost, quality and access to services (Gül, Güneri \& Güneş, 2017).

The three main items to consider in the choice of a hospital are quality, cost and access to services. The patients consider an ideal hospital as one that provides satisfactory services with lower costs and easy access to services. The emergency ward is a sub unit in the hospital which is a major access point where from the beginning patients are given treatments for a wide range of sicknesses and injuries. These sicknesses and injuries may be deadly and requires prompt attention (McLaughlin \& Hayes, 2008; Gül, Güneri \& Güneş, 2017). Therefore, any delay in patient treatment time in the emergency ward may result in undesired consequences and loss of lives.

A major determinant of efficient service delivery in hospitals, especially, the emergency ward is the time required for treatment of patients (Gül, Güneri \& Güneş, 2017). The patient flow procedure in the emergency ward of Aminu Kano teaching hospital (AKTH) is in the following order: walk in patient or wheel in patient, assessment and intervention by the triage personnel, registration, and clinical review by doctor. After the clinical review, the patient may be discharged, or kept for observation for 12 hours or less, or may be admitted in emergency ward for 48 hours. This is followed by sending the patient to available unit, or shifting patient to another facility, or discharged. After discharged of the patient, he or she may be given health education or sent for pharmaceutical processes, then followed by account disengagement and billing.

Different problems arise due to complexity and uncertainties involved in the listed activities performed in the emergency ward. This naturally leads to unnecessary delays in receiving treatments, poor resources utilization, and improper staff scheduling. Some of the reasons for prolonged waiting times in emergency ward are long waits to see the doctor, delay in assessment and intervention by the triage personnel, delays in carrying out tests and getting the results, and shortage of medical staff (McLaughlin \& Hayes, 2008; Gül, Güneri \& Güneş, 2017). Therefore, changes must be made to improve performance and encourage efficient patient flow processes of 
the emergency ward. Some of the needed changes are to get rid of redundant activities and identifying alternative flow processes. Luckily, two closely related operations research techniques, critical path method (CPM) and program evaluation and review technique (PERT), are readily available for use of managers of the emergency wards to assist them in carrying out these responsibilities. These techniques heavily rely on the application of project network diagrams in planning and displaying the coordination of all the activities (Hillier \& Lieberman, 2001).

Szmer \& Węgrzyn (2019) deduced that the efficiency of the operating ward has a great effect on the financial condition of a hospital. The operating department is estimated to provide two-thirds of the income, at the same time generate $40 \%$ of the costs. A set back in the implementation of the operation plans may results in financial losses for the whole hospital. The work focused on how to eliminate or at least reduced these delays in the operating department. Furthermore, the study had shown how efficient time management and application of other corrective measures had reduced the delays by $16 \%$.

Gül, Güneri \& Güneş (2017) identified activities affecting patient flow through the emergency department of a university teaching hospital in Turkey. Project network was drawn using fuzzy activity times obtained from the emergency department of the university hospital. Fuzzy CPM and fuzzy PERT were used to calculate the critical path, expected time, and float times for the project completion.

Jain (2013) used PERT and CPM to monitor and control health related programmes and projects. It was shown that the methods enhance the calculation of the expected projects completion time, identify the tasks and the start and finish times of activities. Furthermore, the work analyzed the impact of revising and rescheduling of the projects, delay of some activities, estimation of the resources required to complete each activity and determination of the type of extra resources needed.

Merten (1966) shows that PERT can be of great assistance to the public health manager in the precise evaluation of project progress, harmonization, logical eradication of redundant activities, allotting of responsibility for different components of the project, as well as initial and final evaluation. PERT can also be used for multiple screening, other public health projects, budget 
and personnel employment processes. In addition, the technique could be profitably applied to projects such as organizing different kinds of large conferences, R \& D programs, immunization programs, acquiring and processing of data for essential records, and the promotion of law making (Merten, 1966).

The aim of this paper is to evaluate the patients flow in the emergency ward of AKTH using fuzzy CPM (FCPM) and fuzzy PERT (FPERT). The tools and methods used in this research are aimed at providing time and cost-effective scheduling process so as to improve patients flow. In particular, these improvements will greatly reduce delays in the duration of treatment of patients.

\section{METHODOLOGY}

In this section, the methodology used in this paper is described. First, the classical CPM and PERT Algorithms are discussed, and then the FCPM and FPERT Algorithms are also described.

\subsection{CPM and PERT Algorithms}

Project network is a graphical representation of logical and sequentially connected activities and events of a project. The project network is sometimes called arrow diagram. The two most widely applied network techniques are PERT and CPM. PERT and CPM are useful for planning, scheduling and executing large time-bound projects which involve careful coordination of a variety of complex and interrelated activities and resources.

Essentially, there are six steps which are common to both the techniques that are described below.

1) Specify the individual activities and milestones. These activities are jobs needed for the completion of a project while, milestones are significant events marking the beginning and the end of one or more activities.

2) Develop a logical sequence of those activities. Some of these activities are dependent upon the completion of others. The determination of the predecessor-successor relationships between the various activities of the project is useful in the construction of the network diagram. In some cases, these activities may require detailed analysis to determine the precise order in which they must be arranged. The activities are normally arranged in tabular form. 
3) Construct a network diagram. The network diagram can now be drawn using activity sequence information. A node represents each activity in the network, while the arrow indicates the relationship between activities (see, fig. 1) and general progression in time. There exist some software packages that simplify this step by automatically converting tabular activity information into a network diagram (Hillier \& Lieberman, 2001).

4) Determine the completion time for individual activity. Estimation of the time needed to complete each activity is done using past experience or the estimates of knowledgeable persons. Units of time for activity completion in common use are minutes, hours, weeks, days etc.

CPM is a deterministic model that does not take into account variation in the completion time, so only one number can be used to determine an activity time (Punmia \& Khandelwal, 2006). In contrast, PERT is a probabilistic model and has the ability to deal with uncertainty in activity completion time. The classical PERT model usually has three time estimates (.i.e., Optimistic time, most likely time and pessimistic time).

PERT uses a beta probability distribution for the time estimates and the expected time for each activity can be calculated using (1).

$$
t_{e}=\frac{t_{o}+4 t_{m}+t_{p}}{6}
$$

where, $t_{e}=$ expected time, $t_{o}=$ optimistic time, $t_{m}=$ most likely time, and $t_{p}=$ pessimistic time.

According to Punmia \& Khandelwal (2006), the estimated expected time for the completion of each activity is usually displayed on a network diagram.

To calculate the variance for each activity completion time, if three values are selected for standard deviation times each for the optimistic and pessimistic times; it means there are six standard deviations between them. The variance is calculated using (2).

$$
\sigma^{2}=\left(\frac{t_{p}-t_{o}}{6}\right)^{2}
$$

5) Identify the critical path. The critical path is the path with the longest duration through the network. It is calculated by adding all the activity time in each sequence and identify the longest 
path in the project. The critical path determines the total calendar time required for the project. The significance of the critical path is that the activities that lie on it cannot be delayed without delaying the project. As a result of its impact on the entire project, critical path analysis is an important aspect of project planning. If activities outside the critical path speed up or slow down (within limits), the total project time does not change. The amount of time that a non - critical path activity can be delayed without affecting the project is referred to as a slack time. Therefore, the critical path is the path in the network in which none of the activities have slack.

The critical path can easily be identified by determining the following four parameters for each activity:

$$
\begin{array}{cl}
\text { i. } & \text { ES - Earliest Start time } \\
\text { ii. } & \text { EF - Earliest Finish time } \\
\text { iii. } & \text { LS - Latest Start time } \\
\text { iv. } & \text { LF - Latest Finish time }
\end{array}
$$

These times are calculated using the expected time for the relevant activities. The earliest start time is the earliest time at which an activity can begin given that its predecessor activities must be completed first. The earliest finish time is the same as the earliest start time for the activity plus the time required to complete the activity. The ES and EF are determined by working forward through the network. The latest finish time is the latest time at which an activity can be completed without delaying the project. The latest start time, equal to the latest finish time minus the time required to complete the activity. LS and LF are found by working backward through the network.

6) Update the network diagram

As the project progresses, the actual activity completion times will be ascertained and the network diagram can be updated to include this actual times. A new critical path may emerge, and structural changes may be made in the network if project requirements change. In situations where delays are visible, additional resources may be needed to be within schedule time and therefore the network diagram has to be changed to reflect the new situation.

\subsection{FCPM and FPERT Algorithms}


The notations and process steps of FCPM and FPERT are adopted from (Omer Atli \& Cengiz Kahraman, 2012; Gül, Güneri \& Güneş, 2017) as follows:

\section{Notations}

$\mathrm{N}$ : All nodes in a project network,

$A_{\mathrm{ij}}$ : The activity between two nodes (from $\mathrm{i}$ to $\mathrm{j}$ )

FAT $_{\mathrm{ij}}$ : Fuzzy activity time of $\mathrm{A}_{\mathrm{ij}}$

EFT: Earliest fuzzy time

LFT: Latest fuzzy time

LFT: Latest fuzzy time

$S F T_{i j}:$ The total fuzzy slack time of $A_{i j}$

$\operatorname{FCPM}\left(\mathrm{P}_{n}\right)$ : The fuzzy completion time of path $\mathrm{P}_{n}$.

$\mathrm{t}$ : Number of activities in a project network

$S(j)$ : The set of all activities after node $j$.

NS(j): The set of all nodes connected to all activities after node $\mathrm{j}=\left\{k / A_{j k} \in S(j), \mathrm{k} \in \mathrm{N}\right\}$

$P(j)$ : The set of all activities before node $\mathrm{j}$.

$\mathrm{NP}(\mathrm{j})$ : The set of all nodes connected to all activities before node $\mathrm{j}=\left\{\mathrm{i} / A_{i j} \in P(j), \mathrm{i} \in \mathrm{N}\right\}$

\subsubsection{Steps for determination of FCPM}

Step 1: Set initial node $E F T_{1}=(0,0,0,0)$.

Step 2: Evaluate $\beta$, the risk factor for each $A_{i j}$ using (3). 


$$
\beta=\sum_{i} \sum_{j}\left(\frac{\left(b_{i j}-a_{i j}\right)}{\left(b_{i j}-a_{i j}\right)+\left(\mathrm{d}_{i j}-c_{i j}\right)}\right) / t
$$

The risk situation is neutral, if $\beta=0.5$. If $\beta<0.5$ it is risky and a more risky situation if $\beta>0.5$

Step 3: Determine the ETF for individual node using (4).

$$
E F T_{j}=E F T_{i} \oplus F A T_{i j}
$$

Step 4: Take $E F T_{j}$ with the highest value for each node after comparing the $E F T_{j} \mathrm{~s}$ for intersecting nodes using (5).

$$
E F T_{j}=\max \left\{E F T_{i} \oplus F A T_{i j}\right\}=\max \left\{\left(a_{x}, b_{x}, c_{x}, d_{x}\right),\left(a_{y}, b_{y}, c_{y}, d_{y}\right)\right\}
$$

Step 4(a): Evaluate the values of $x_{1}$ and $x_{2}$ using (6)

$$
x_{1}=\min \left\{a_{x}, b_{x}, c_{x}, d_{x}, a_{y}, b_{y}, c_{y}, d_{y}\right\}, x_{2}=\max \left\{a_{x}, b_{x}, c_{x}, d_{x}, a_{y}, b_{y}, c_{y}, d_{y}\right\}
$$

Step 4(b): Determine the values of $\mathrm{R}\left(\left(a_{x}, b_{x}, c_{x}, d_{x}\right)\right)$ and $\mathrm{R}\left(\left(a_{y}, b_{y}, c_{y}, d_{y}\right)\right)$ using (7)

$$
\mathrm{R}\left(\left(a_{i}, b_{i}, c_{i}, d_{i}\right)\right)=\beta\left[\left(\mathrm{d}_{i}-x_{i}\right) /\left(\mathrm{x}_{2}-\mathrm{x}_{1}-\mathrm{c}_{i}+\mathrm{d}_{i}\right)\right]+(1-\beta)\left[1-\left(\mathrm{x}_{2}-\mathrm{a}_{i}\right) /\left(\mathrm{x}_{2}-\mathrm{x}_{1}+\mathrm{b}_{i}-\mathrm{a}_{i}\right)\right]
$$

Step 4(c): Rank the results of $\mathrm{R}\left(\left(a_{i}, b_{i}, c_{i}, d_{i}\right)\right)$ and take the one that is bigger.

Step 5: Determine LFT for individual node using (8)

$$
L F T_{j}=E F T_{k} \Theta F A T_{j k}
$$

Step 6: Consider $L F T_{j}$ with the lowest value for each node after comparing the $L F T_{j} s$ for intersecting nodes using (9)

$$
L F T_{j}=\min \left\{E F T_{k} \Theta F A T_{j k}\right\}=\min \left\{\left(a_{x}, b_{x}, c_{x}, d_{x}\right),\left(a_{y}, b_{y}, c_{y}, d_{y}\right)\right\}
$$

Note that the sub-steps of Step 6 are similar to that of step 4.

Step 7: Determine SFT for every activity using (10) 


$$
S F T_{i j}=L F T_{j} \Theta\left(E F T_{i} \oplus F A T_{i j}\right)
$$

Step 8: Determine all possible paths and find the FCPM values for all paths. FCPM can be calculated using (11).

$$
\operatorname{FCPM}\left(P_{n}\right)=\sum S F T_{i j}
$$

Step 9: Consider all FCPMs and take the minimum value as the FCPM as done in Step 6 using (12). Rank the results of $\mathrm{R}\left(\left(a_{i}, b_{i}, c_{i}, d_{i}\right)\right)$ and take the one that has smaller value.

$$
\operatorname{FCPM}\left(P_{n}\right)=\min \left\{\operatorname{FCPM}\left(P_{i}\right) \mid \mathrm{i}=1,2,3, \ldots, \mathrm{n}\right\}
$$

Step 10: Consider the path with the smallest FCPM and take it as the critical path.

\subsubsection{Steps for determination of FPERT}

To determine the FPERT requires finding a generalized mean and calculating the standard deviation based on trapezoidal fuzzy numbers. Generalized mean can be calculated using (14).

$$
\mathrm{M}\left(a_{1}, b_{1}, c_{1}, d_{1}\right)=\frac{a_{1}+b_{1}+c_{1}+d_{1}}{4}
$$

The standard deviation for the trapezoidal fuzzy numbers can be calculated using (15).

$\mathrm{D}\left(a_{1}, b_{1}, c_{1}, d_{1}\right)=\frac{\left[3\left(a_{1}^{2}+b_{1}^{2}+c_{1}^{2}+d_{1}^{2}\right)-2\left(a_{1} b_{1}+a_{1} c_{1}+b_{1} c_{1}+a_{1} d_{1}+b_{1} d_{1}+c_{1} d_{1}\right)\right]}{36}$

After the critical path is determined, the next thing is to find the probability of meeting the scheduled completion time of a patient treatment. When activities of the project are large, as with the PERT projects then the expected time of individual activities has random probability distribution and the variation of the expected time for the project is completely assumed to have a normal distribution. This assumption of normal probability distribution is based on central limit theorem (Punmia \& Khandelwal, 2006).

The probability factor $(Z)$ is called normal deviate which is the distance from the mean expressed in terms of standard deviation $\sigma$ (Punmia \& Khandelwal, 2006). Z may be positive, zero or 
negative. If $Z>0$, then the probability of completing a project in time is more than 0.5 , if $Z=0$, then the probability of completing the project in time is 0.5 and if $Z<0$ then the probability of completing the project in time is less than 0.5 .

The mean and standard deviation of the normal probability distribution are used for the calculation of $\mathrm{Z}$ using relation (16).

$$
Z=\frac{X-\mu}{\sigma}
$$

\section{A CASE STUDY}

A case study is performed in the emergency ward of Aminu Kano teaching hospital (AKTH), Kano, Nigeria. The activities and sequence of patients flow in the emergency department is shown on table 1. FATs in respect of each activity in the treatment of patient in emergency ward is shown on table 2, while the project network chart for the treatment of patient is shown in figure 1 .

Table 1. Activities for the Treatment of Patient in Emergency Department

\begin{tabular}{|c|l|}
\hline Symbol & \\
\hline A & Moving of the patient from ambulance or private car to emergency ward \\
\hline B & Walk in patient or wheel in patient \\
\hline C & Assessment and intervention by the triage personal \\
\hline D & Registration \\
\hline E & Clinical Review \\
\hline F & $\begin{array}{l}\text { Sending the patient to available unit in the hospital determined by the severity of } \\
\text { illness }\end{array}$ \\
\hline G & Change the patient to a new facility \\
\hline
\end{tabular}




\begin{tabular}{|c|l|}
\hline $\mathrm{H}$ & Discharge \\
\hline $\mathrm{I}$ & Receive bill for services rendered \\
\hline
\end{tabular}

Table 2. FATs for every activity for treatment of patient in emergency ward

\begin{tabular}{|lccccc|}
\hline & & & & \\
Activity & Predecessor & \multicolumn{5}{c|}{ FATs (in minutes) } \\
& & & & \\
\hline A & - & 3 & 4 & 4.5 & 5 \\
\hline B & A & 0.5 & 1 & 1.5 & 2 \\
\hline C & B & 0.5 & 1 & 2 & 4 \\
\hline D & C & 10 & 12 & 15 & 30 \\
\hline E & D & 10 & 20 & 30 & 40 \\
\hline F & E & 440 & 1920 & 2400 & 2880 \\
\hline G & E & 30 & 40 & 60 & 90 \\
\hline H & F,G & 30 & 60 & 90 & 120 \\
\hline I & H & 30 & 45 & 120 & 180 \\
& & & & & \\
\hline
\end{tabular}

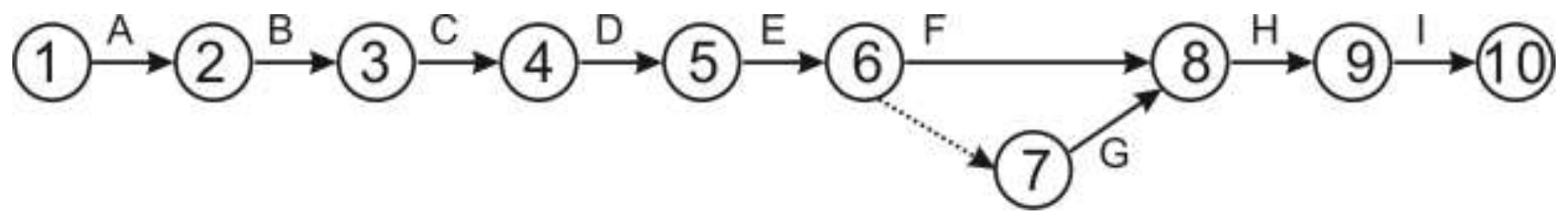

Figure 1: Project Network Chart for the Treatment of Patient in Emergency Ward 
In this work, trapezoidal fuzzy numbers are used to represent the fuzzy activity times. The $a_{i j}$ and $d_{i j}$ are the lowest and highest values of evaluating activity time for $A_{i j}$, whereas $b_{i j}$ and $c_{i j}$ represent the first and third quartile respectively of activity time for $A_{i j}$. Suppose that only one set of four data; $a_{i j}, b_{i j}, c_{i j}$ and $d_{i j}$ is obtained, and then this data can be arranged from the lowest to highest. If on the other hand, no information is obtained in respect of activity $A_{i j}$, then the $F A T_{i j}=\left(a_{i j}, b_{i j}, c_{i j}, d_{i j}\right)$ will be determined subjectively by the manager of the emergency ward based on his or her experience.

\section{Computations of EFT and LFT Values}

The values of EFT and LFT are calculated for each node by using appropriately equations (8), (9), (10), (11) and (12). The results of these computations are shown on table 3 and table 4.

Table 3. EFT Values

\begin{tabular}{l|cccc}
\hline NODE & \multicolumn{5}{|c}{ EFT Values } \\
\hline 1 & 0 & 0 & 0 & 0 \\
\hline 2 & 3 & 4 & 4.5 & 5 \\
\hline 3 & 3.5 & 5 & 6 & 7 \\
\hline 4 & 4 & 6 & 8 & 11 \\
\hline 5 & 14 & 18 & 23 & 41 \\
\hline 6 & 24 & 38 & 53 & 81 \\
\hline 7 & 24 & 38 & 53 & 81 \\
\hline 8 & 464 & 1958 & 2453 & 2961 \\
\hline 9 & 494 & 2018 & 2543 & 3081 \\
\hline 10 & 524 & 2063 & 2663 & 3261 \\
\hline & & & & \\
\hline
\end{tabular}


Table 4. LFT Values

\begin{tabular}{l|cccc}
\hline NODES & \multicolumn{5}{|c}{ LFT Values } \\
\hline 1 & -2737 & -600 & 600 & 2737 \\
\hline 2 & -2732 & -595.5 & 604 & 2740 \\
\hline 3 & -2730 & -594 & 605 & 2740.5 \\
\hline 4 & -2726 & -592 & 606 & 2741 \\
\hline 5 & & & & \\
\hline 6 & -2696 & -577 & 618 & 2751 \\
\hline 7 & -2656 & -547 & 638 & 2761 \\
& & & & \\
\hline 8 & -2656 & -547 & 638 & 2761 \\
\hline 9 & 224 & 1853 & 2558 & 3201 \\
\hline 10 & 344 & 1943 & 2618 & 3231 \\
\hline
\end{tabular}

\section{Computations of SFT Values}

The total SFTs are calculated for each activity using (12), the results of the calculations are shown on table 5 .

Table 5. SFT Values

\begin{tabular}{|c|llll}
\hline SFT & \multicolumn{5}{|c}{ VALUES } \\
\hline $1-2$ & -2737 & -600 & 600 & 2737 \\
\hline $2-3$ & -2737 & -600 & 600 & 2737 \\
& & & & \\
\hline $3-4$ & -2737 & -600 & 600 & 2737 \\
& & & & \\
\hline $4-5$ & -2737 & -600 & 600 & 2737 \\
& & & & \\
\hline
\end{tabular}




\begin{tabular}{|l|llll}
\hline $5-6$ & -2737 & -600 & 600 & 2737 \\
\hline $6-7$ & -2737 & -600 & 600 & 2737 \\
\hline $6-8$ & -2737 & -600 & 600 & 2737 \\
\hline $7-8$ & 53 & 1740 & 2480 & 3147 \\
\hline $8-9$ & -2737 & -600 & 600 & 2737 \\
\hline $9-10$ & -2737 & -600 & 600 & 2737 \\
& & & & \\
\hline
\end{tabular}

\section{Determination of Critical Paths}

When all possible paths are found, then FCPMs for each node are evaluated using (11). The results are compared and FCPM $\left(\mathrm{P}_{\mathrm{i}}\right)$ is obtained using (3) in Step 4. The calculated values for FCPM and R[FCPM $\left.\left(\mathrm{P}_{\mathrm{i}}\right)\right]$ of every path obtained are presented on table 6 .

Table 6. FCPM AND R [FCPM $\left.\left(P_{i}\right)\right]$ values of each possible path

\begin{tabular}{|rr|rrrr|r|}
\hline SN & Possible paths & \multicolumn{3}{|c|}{$\operatorname{FCPM}\left(P_{i}\right)$} & R \\
\hline 1 & $1-2-3-4-5-6-7-8-9-10$ & -21843 & -3060 & 7280 & 25043 & 0.4902 \\
\hline 2 & & & & & & \\
\hline
\end{tabular}

It can be seen from table 6 that the critical path is $1-2-3-4-5-6-8-9-10$, since $R$ (FCPM $\left.\left(P_{2}\right)\right)$ has the lowest value of all possible paths. Therefore, it can be concluded that the length of treatment time lies approximately between 2063 and 2663 minutes i.e. (524, 2063, $2663,3261)$.

\section{Determination of FPERT}

In the determination of FPERT, the computation of the generalized mean value for each activity can be done using (14) as shown on table 7 . 
Table 7. Mean for each activity

\begin{tabular}{|c|c|c|c|c|c|}
\hline Activity & $\mathrm{A}$ & B & $\mathrm{C}$ & D & Mean \\
\hline $\bar{A}$ & 3 & 4 & 4.5 & 5 & 4.1250 \\
\hline B & 0.5 & 1 & 1.5 & 2 & 1.2500 \\
\hline $\mathrm{C}$ & 0.5 & 1 & 2 & 4 & 1.8750 \\
\hline $\mathrm{D}$ & 10 & 12 & 15 & 30 & 16.7500 \\
\hline $\mathrm{E}$ & 10 & 20 & 30 & 40 & 25.0000 \\
\hline $\mathrm{F}$ & 440 & 1920 & 2400 & 2880 & 1910.0000 \\
\hline G & 30 & 40 & 60 & 90 & 55.0000 \\
\hline $\mathrm{H}$ & 30 & 60 & 90 & 120 & 75.0000 \\
\hline I & 30 & 45 & 120 & 180 & 93.7500 \\
\hline Total & & & & & 2089.0000 \\
\hline $\begin{array}{l}\text { Activities } \\
\text { of critical path }\end{array}$ & & & & & 2034.0000 \\
\hline
\end{tabular}

Table 8. Variance for each activity

\begin{tabular}{|c|c|c|c|c|c|}
\hline Activity & A & B & C & D & Variance \\
\hline A & 3 & 4 & 4.5 & 5 & 0.2431 \\
\hline B & 0.5 & 1 & 1.5 & 2 & 0.1389 \\
\hline C & 0.5 & 1 & 2 & 4 & 0.7986 \\
\hline D & 10 & 12 & 15 & 30 & 27.4167 \\
\hline E & 10 & 20 & 30 & 40 & 55.5556 \\
\hline
\end{tabular}




\begin{tabular}{|c|r|r|r|r|c|}
\hline F & 440 & 1920 & 2400 & 2880 & 371333.3333 \\
\hline $\mathrm{G}$ & 30 & 40 & 60 & 90 & 233.3333 \\
\hline $\mathrm{H}$ & 30 & 60 & 90 & 120 & 500.0000 \\
\hline I & 30 & $45 \mathrm{~s}$ & 120 & 180 & 1618.7500 \\
\hline Total & & & & & 373769.5695 \\
\hline $\begin{array}{c}\text { Activities } \\
\text { of critical } \\
\text { path }\end{array}$ & & & & & 373536.2362 \\
\hline
\end{tabular}

Computation of standard deviation with sum of variance of activities on the critical path is performed and the result is

$$
\sigma=\left(\sum \sigma_{i j}^{2}\right)^{\frac{1}{2}}=(373536.2362)^{\frac{1}{2}}=611.1761
$$

Computation of expected completion time with sum of mean of activities on the critical path gives

$$
\mu=\sum m_{i j}=2034
$$

The calculated values of mean and standard deviation of the normal probability distribution are used to determine various values of $\mathrm{Z}$ using relation (16) and the results are shown on table 9.

Table 9. Some probabilities of the project completion time

\begin{tabular}{|c|c|c|}
\hline $\mathrm{X}$ & Z Values & Probability \\
\hline$<1800$ & -0.3829 & 0.3520 \\
\hline$<2000$ & -0.0556 & 0.4761 \\
\hline & & \\
\hline
\end{tabular}




\begin{tabular}{|r|c|c|}
\hline$<2200$ & & 0.6064 \\
\hline$<2400$ & 0.5988 & 0.7257 \\
\hline$<2600$ & 0.9260 & 0.8238 \\
\hline$<2800$ & 1.2533 & 0.8944 \\
\hline$<3000$ & 1.5806 & 0.9429 \\
\hline$<3200$ & & 0.9719 \\
\hline$<3400$ & 1.9078 & 0.9875 \\
\hline$<3600$ & & 0.9948 \\
\hline
\end{tabular}

\section{Discussion of results}

The Case study describes a patient treatment project which includes 9 subactivities as shown on Table 1.The details of the activities and their predecessor relationships are presented in column 1 and 2 of Table 2 while, the details of the activities and time estimates of activity durations are also shown in columns 3, 4, 5 and 6 of table 2 .

It was found that the critical path is $1-2-3-4-5-6-8-9-10$, since $\mathrm{R}\left(\mathrm{FCPM}\left(P_{2}\right)\right)$ has the lowest value of all possible paths. Therefore, it can be concluded that the length of treatment time lies approximately between 2063 and 2663 minutes.

The critical path was found to consist of the following activities: A (Moving of the patient from ambulance or private car to emergency ward), B (Walk in the patient or wheel in the patient to the ward), $\mathrm{C}$ (Assessment and intervention by the triage personal), D (Registration), E (Clinical Review), F (Sending the patient to available unit in the hospital depending on the severity of illness), H (Discharge) and I (Receive bill for services rendered). 
Punmia and Khandelwal (2006) established that the critical path does not allow any flexibility in critical activities. As a change in any of the critical activities A, B, C, D, E, F, H or I on the critical path will negatively affect the entire treatment time of patients in the emergency ward.

Table 9 gives the values of $Z$, with some probabilities of the treatment completion time of the patient. As noted in the first and last columns, the probability of completing the treatment of the patient in less than 1800 minutes is $35.2 \%$, in less than 2000 minutes is $47.61 \%$, in less than 2200 minutes is $60.64 \%$, in less than 2400 minutes is $72.57 \%$, in less than 2600 minutes is $82.38 \%$, in less than 2800 minutes is $89.44 \%$, in less than 3000 is $94.29 \%$, in less than 3200 is $97.19 \%$, in less than 3400 minutes is $98.75 \%$, and in less than 3600 minutes is $99.48 \%$.

It is observed that the probability of completing the treatment of the patient in less than 2200 minutes $(60.64 \%)$ and in less than 2800 minutes $(89.44 \%)$ is within the probable range. This was found to be approximately between 2063 and 2663 minutes through the use of FCPM. However, the treatment will be completed before the 3600th minutes, even after taking into consideration of the worst case scenario.

\section{Conclusion}

This paper presents the patients treatment evaluation project scheduling in AKTH using CPM and PERT techniques. These techniques provide both the deterministic and probabilistic models used in the planning, scheduling and controlling the patients' treatment durations.

Project network analysis had been used as a major tool to efficiently analyze the number of minutes taken to complete the treatment of a patient in the emergency department of AKTH. This is done to significantly reduce delays in the duration of treatments, weed out redundant activities at the same time increase the quality of treatment of patients. The effectiveness of the emergency ward will largely depends on the skillful management of time in relation to patient flow processes.

In the evaluation of patient flow of the emergency department, PERT method was used to check the Pessimistic Time (longest time possible and can be seen as usual delay), Most Likely Time (the time the activity would often require if normal conditions prevailed) and Optimistic Time 
(shortest time possible if things go perfectly) as well as the probability of completing the treatments within a specific time.

\section{References}

Gül, M., Güneri, A. F. \& Güneş, G. (2017). Project Management in Healthcare: A Case Study for Patient Flow Evaluation in an Emergency Room using Fuzzy CPM AND Fuzzy PERT. Sigma J. Eng \& Nat Sci 8 (1), 41-51.

Hillier, F.S., \& Lieberman, G. J. (2001). Introduction to operations research, McGraw-Hill Series in Industrial Engineering and Management Science, 468-533.

Jain, S. (2013). Application of PERT Technique in Health Programme Monitoring and Control. International Journal of Research in Commerce, IT \& Management. Volume No. 3, Issue No. 04 (April), ISSN 2231-5756. http://ijrcm.org.in/

McLaughlin, D. B. \& Hays, J.M. (2008). Healthcare operations management. Health Administration Press, Chicago AUPHA Press, Washington, DC.

Merten, W. (1966). PERT and Planning for Health Programs. Public Health Report. Vol. 81, No. 5 .

Omer Atli \& Cengiz Kahraman (2012): Aircraft Maintenance Planning Using Fuzzy Critical Path Analysis, International Journal of Computational Intelligence Systems, 5:3, 553-567.

Punmia, B. C. \& Khandelwal, K. K. (2006). Project planning and control with PERT and CPM, Laxmi Publications (P) Ltd, New Delhi

Reid, R. D. \& Sanders, N. R. (2010). Operations Management, $4{ }^{\text {th }}$ Edition, Wiley.

Szmer, A. S. \& Węgrzyn, V. (2019). Reducing Delays in the Operation Plan of a Hospital: The Direct Observation Approach. International Journal for Quality Research 14(2) 471-486 ISSN 1800-6450. 